\title{
AVALIAÇÃO COGNITIVA E MOTORA EM IDOSAS COM DOENÇA DE
}

\author{
ALZHEIMER
}

Wiviane Maria Torres de Matos FREITAS ${ }^{1}$

Larissa Alves WANZELER ${ }^{2}$

Evellin dos Santos TEIXEIRA ${ }^{3}$

\begin{abstract}
${ }^{1}$ Fisioterapeuta, Mestre em Doenças Tropicais pela Universidade Federal do Pará, Especialista em Geriatria e Gerontologia e Docente do curso de Fisioterapia do Centro Universitário do Pará. wivianematos@ yahoo.com.br

${ }^{2}$ Graduada no curso de Bacharelado em Fisioterapia do Centro Universitário do Pará. Larissawanzeler@ yahoo.com.br

${ }^{3}$ Graduada no curso de Bacharelado em Fisioterapia do Centro Universitário do Pará. evellinsteixeira@gmail.com.
\end{abstract}

Recebido em: 22/06/2015 - Aprovado em: 09/01/2016 - Disponibilizado em: 30/07/2016

\begin{abstract}
RESUMO
Introdução: A doença de Alzheimer é caracterizada por déficits progressivos de diversas funções cognitivas, e existe também o comprometimento motor pouco falado, mas que interfere na funcionalidadedo idoso envolve habilidades aeróbicas do individuo, como a força, flexibilidade, coordenação motora e agilidade. Objetivo: Avaliar a cognição e aptidão motora de idosas com Doença de Alzheimer de uma Instituição para Idosos. Material e Método: A pesquisa se iniciou após aprovação do Comitê de Ética e assinatura do TCLE. As idosas da instituição responderam a uma ficha para perfil sócio demográfico e posteriormente foram avaliadas através do Mini Exame do Estado Mental (MEEM) e da Escala Motora para Terceira Idade.Resultados: Participam deste estudo 10 mulheres idosas com diagnóstico de doença de Alzheimer com idade média de 78,8 anos. Apresentaram média de 19.8 pontos no MEEM, quando comparado ao tempo da doença, encaixavam-se na classificação de comprometimento intermediáriodo estado cognitivo. Quanto à aptidão motora, a maior parte das idosas (60\%) apresentou rendimento normal médio, sendo assim, as idosas ainda não têm comprometimento significativo na função motora. Entretanto, destaca-se que o menor desempenho foi na avaliação de AM3 (movimentos de estabilidade corporal e propriocepção) dessas idosas com DA. Conclusão:O estudo conclui que as idosasfrequentadoras da instituição, não apresentam comprometimentos significativos nem na função cognitiva, nem na função motora, fato esse associado ao estimulo recebido frequentemente. Desta maneira pode-se supor que com o maior número de estímulos oferecidos as idosas mais tardiamente, irão apresentar declínios funcionais.
\end{abstract}

Palavras-chave: Doença de Alzheimer. Idoso.Cognição.Atividade Motora. Avaliação em Saúde.

\section{COGNITIVE AND MOTOR ASSESSMENT IN ELDERLY WITH ALZHEIMER'S DISEASE}

\begin{abstract}
Introduction: Alzheimer's diseaseis characterized byprogressivedeficitsin variouscognitive functions, and there ismotor impairment, littletalked about butthat interferes with thefunctionalityof the elderlyinvolvesaerobicabilitiesof the individual, as well as strength, flexibility, coordination and agility. Objective:Assess cognition and motor fitness of elderly with Alzheimer's disease in the institution for Seniors.Material and Method:The research was initiated after approval of the Ethics Committee and signed the consent form. The older the institution responded to a record for sociodemographic profile and were subsequently evaluated using the Mini Mental State Examination (MMSE), and Motor Scale for the Elderly. Results:Participating in this study, 10 elderly women with a diagnosis of Alzheimer's disease with a mean age of 78.8 years.Had a mean MMSE 19.8, compared to the timeofdisease withcognitiveimpairmentintermediatestate. Asthe drivingfitness,mostwomen $(60 \%)$ had normal returnmedium, so olderstill havesignificant impairmentin motor function.However, it is noteworthy thatthelowest performancein the evaluation ofAM3(movements of bodystability andproprioception) of theseelderlywith AD.Conclusion:The studyconcludesthat elderlywith ADRenewthe institution, do not exhibit significantimpairmentorcognitive functionormotor function, a fact associated with thestimulusreceivedby them. Thus, one can assumethat with thegreater number ofstimuliofferedthe elderly, will presentlaterfunctional decline.
\end{abstract}

Key words: Alzheimer's disease; Seniors; Cognition; Motor Activity.Health Evaluation. 


\section{INTRODUÇÃO}

O envelhecimento populacional é uma realidade mundial. Em países desenvolvidos a população idosa já ultrapassou o número de crianças, e estima-se que em 2050 teremos $32 \%$ de pessoas idosas, totalizando a proporção de uma criança para cada dois idosos(LUCCHETTI ET AL, 2011).

A velhice é caracterizada por mudanças biológicas, psicológicas, cognitivas e sociais que aumentam a predisposição a situações de incapacidade funcional, multimorbidade e aumento do risco a situações de vulnerabilidade(PERRACINI E FLO, 2013).

Sob essa perspectiva, o declínio funcional cognitivo esta associado a uma doença frequente do envelhecimento, a doença de Alzheimer (DA). Tal doença já representa um problema de saúde publica em razão do envelhecimento populacional (APRAHAMIAN, MARTINELLI E YASSUDA, 2008).Atualmente as demências atingem 20\% das pessoas na América Latina e no Brasil 38,9\%, estima-se que no ano de 2025, 34 milhões de pessoas vão ter um quadro demencial, e uma grande proporção delas terá DA(MEDEIROS E RO, 2009; ZIDAN ET AL, 2012).

A doença de Alzheimer caracteriza-se por uma neurodegeneração que afeta circuitos nervosos cerebrais, processo progressivo que afeta a memoria recente, como principal agressão a função cognitiva, além de interferir no humor e estado comportamental (FLORINDA ET AL, 2003).

O prejuízo cognitivo é a principal queixa clínica do paciente com a Doença de Alzheimer(GALLO, 2001). A prevalência dessa doença aumenta proporcionalmente com o avanço da idade, sendo este o principal fator de risco, e geralmente os primeiros sintomas da DA aparecem após os 65 anos, de modo que, entre as pessoas com mais de 85 anos, 20\% apresentam uma forma grave da doença(APRAHAMIAN, MARTINELLI E YASSUDA, 2008; PERRACINI E FLO, 2013).

O estágio da doença é classificado em: Estágio inicial - descrito pela dificuldade em pensar com clareza, situações de declínio em seu rendimento funcional de tarefas complexas; Estágio intermediário - onde os sujeitos apresentam dificuldades para desempenhar tarefas mais simples, como utilizar utensílios domésticos e cuidar da própria higiene e alimentar-se; Estágio avançado - o mais incapacitante de realizar qualquer atividade de modo independente(ABREU, 2005).

Diferentemente do que se imagina, a DA não repercute apenas na função cognitiva, é uma doença que também gera limitações motoras. O comprometimento da aptidão funcional ou motora no envelhecimento ou particularmente na DA, não depende exclusivamente da capacidade aeróbia do individuo, e sim também de outros 
componentes como força, flexibilidade, coordenação motora e agilidade (ZAGO E GOBBI, 2003).

A doença de Alzheimer ocasiona uma perda progressiva da autonomia assim como na dependência funcional e necessidade de cuidados específicos.Dentre as limitações funcionais integradas com a doença, destacase a perda das atividades instrumentais ditas como atividades mais complexas tais como cozinhar, transferir-se, o uso de transportes, entre outras, e posteriormente ocasiona a perda na realização das tarefas diárias, tarefasmenos complexas como realizar a higiene pessoal, comer, vestir-se, etc. (ZIDAN ET AL, 2012).

A DA associada ao processo de envelhecimento prejudica o recrutamento e ativação das unidades motoras, desta maneira prejudica o movimento global do idoso, em particular no idoso com doença de Alzheimer (ROSA NETO ET AL, 2004).

A doença de Alzheimer é progressiva, assim como seus efeitos sobre o sistema do indivíduo, em base disso, o estudo buscou avaliar a função cognitiva e aptidão motora de idosas com Doença de Alzheimer de uma instituição em Belém/PA.

\section{MATERIAIS E MÉTODOS}

A presente pesquisa respeitou as Normas de Pesquisa Envolvendo Seres Humanos (Res. CNS 466/12) do Conselho
Nacional de Saúde. Iniciou após a autorizaçãopela Instituiçãopara Idosos selecionada para a pesquisa, no município de Belém, posterior à submissão e aprovação sob o parecer de número 153.106 avaliado pelo Comitê de Ética em pesquisa do Centro Universitário do Pará.

Trata-se de um estudo do tipo observacional e quantitativo, seguindo o delineamento descritivo e transversal.

O grupo foi formado por mulheres idosas devidamente matriculadas na instituição no período da pesquisa. Os critérios de inclusão utilizados na seleção destes indivíduos foram: ser portador de doença de Alzheimer devidamente diagnosticado por médicos para classificação da doença (CID), ter idade igual ou superior a 60 anos, sexo feminino, não poderiam possuir a DA associada a outras doenças crônicodegenerativas, cardiovasculares, reumatológicas ou quaisquer tipo de sequelas motoras que impeçam a realização da pesquisa, e que aceitassem participar espontaneamente da pesquisa.

O estudo excluiu idosas com limitações motoras provocadas por outras doenças associadas ao processo de envelhecimento, cadeirantes ou com uso de outro dispositivo para marcha, idosas com estágio avançado da Doença de Alzheimer que impossibilite a compreensão ou aplicação do trabalho, ou que não quisessem participar da pesquisa. 
Após assinatura do Termo de Consentimento Livre e Esclarecido por meio dos responsáveis legais, as idosas juntamente ao responsável foram submetidas a uma avaliação respondendo a um questionário, de autoria própria, contendo informações comogênero, faixa etária, raça, procedência, escolaridade, profissão/ocupação, tempo de diagnóstico da doença, tempo de matrícula no local de referência, classificação do estágio da DA, facilitando o reconhecimento do perfil das idosas participantes da pesquisa.

Posterior a este primeiro contato, as idosas foram entrevistadas através do Mini Exame do Estado Mental (MEEM) para percepção do estado cognitivo das idosas com DA, tal instrumento contém questões com pontuações especificas por item, que avaliarão aspectos de orientação temporal, orientação espacial, registro de palavras, lembrança, atenção e calculo. O MEEM varia de zero ate uma pontuação máxima de 30 pontos. Estas variáveis serão posteriormente pontuadas e analisadas através do nível de escolaridade edesempenho das respostas. A análise das respostas será em base da avaliação do escore padrão do teste.

Quanto a avaliação da aptidão motora das idosas, o instrumento a ser utilizado é a Escala Motora para Terceira Idade(ROSA NETO ET AL, 2004), que constará de observações e aplicações para a capacidade de aptidão motora geral (AMG), motricidade fina (AM1), motricidade global (AM2), equilíbrio (AM3), esquema corporal (AM4), organização espacial (AM5) e temporal (AM6).

Este método é validado para analise da aptidão geral do individuo e constitui de testes motores clássicos, aplicado individualmente e sua duração é de aproximadamente 30 a 45 minutos. As variáveis analisadas são: AMG, AM1,AM2, AM3, AM4, AM5, AM6, escala motora em 7 (sete) níveis e perfil motor. Passiveis de classificar o nível de aptidão motora que se encontra o individuo submetido ao exame motor.

As idosas foram avaliadas quanto ao aspecto motor, por meio de um circuito de atividades programadas pelas pesquisadoras em parceria com os profissionais da instituição, neste circuito envolviam atividades como dança, pinça fina, andar sobre uma linha reta, etc. atividades capazes de quantificar o rendimento nas variáveis da escala motora para terceira idade.

As variáveis encontradas foram tabuladas em uso do Microsoft Excel 2010 e analisadas permitindo traçar suas medias, medianas, desvio padrão e porcentagens como forma de compreensão e caracterização do perfil dos sujeitos.

\section{RESULTADOS}

Participaram deste estudo 10 idosas com diagnóstico de Doença de Alzheimer, a idade média das pesquisadas foi de 78,8 anos 
\pm 6,72. Todas são naturais do Estado do Pará, e aposentadas. A maioria (40\%) das idosas apresentou o estado civil de casada, no que tange a escolaridade todas referiram o $1^{\circ}$ grau completo/incompleto.

O tempo de diagnóstico da doença das pesquisadas teve média de 1,26 anos $\pm 0,55$, de acordo com a classificação $60 \%$ das idosas está em estágio inicial da doença, as outras $40 \%$ encontram-se em estágio intermediário.

As participantes responderam ao Mini Exame do Estado Mental, como descrito na Tabela I, onde os escores alcançados foram correlacionados ao tempo de diagnóstico da doença.

Tabela 1. Descrição da associação entre o tempo de doença e o comprometimento cognitivo das idosas pesquisadas.

\begin{tabular}{cccc}
\hline IDOSA & $\begin{array}{c}\text { TEMPO DE } \\
\text { DOENÇA } \\
\text { (meses) }\end{array}$ & $\begin{array}{c}\text { RESULTADO } \\
\text { MEEM (0/30) }\end{array}$ & COMPROMETIMENTO \\
\hline ID 1 & 12 & 16 & INTERMEDIÁRIO \\
ID 2 & 12 & 12 & INTERMEDIÁRIO \\
ID 3 & 12 & 30 & INICIAL \\
ID 4 & 12 & 24 & INICIAL \\
ID 5 & 24 & 21 & INICIAL \\
ID 6 & 24 & 22 & INICIAL \\
ID 7 & 12 & 18 & INICIAL \\
ID 8 & 30 & 10 & INTERMEDIÁRIO \\
ID 9 & 12 & 26 & INTERMEDIÁRIO \\
ID 10 & 12 & & INICIAL \\
\hline
\end{tabular}

Fonte: Pesquisa de Campo, 2013.

Após aplicação da Escala Motora, foi possível traçar o perfil destas idosas com doença de Alzheimer, como se observa na Tabela 2. As idosas apresentaram padrões motores abaixo da normalidade nas variáveis AM1, AM2, AM3, AM4 e AM6.
Em especial, houve menor rendimento nas tarefas avaliadas na AM3 (movimento de estabilidade e propriocepção), onde as idosas tiveram dificuldade de realizar as atividades, e obtiveram média de 79,3, padrão inferior da escala motora.

Tabela 2. Análise das variáveis da EMTI das 10 idosas com Doença de Alzheimer. Belém, Pará, Brasil, 2013.

\begin{tabular}{lcccc}
\hline \multicolumn{1}{c}{ VARIÁVEIS } & MÉDIA & DP & MÁXIMO & MÍNIMO \\
\hline AM1 & 93.9 & 12,29 & 109 & 70 \\
AM2 & 86.5 & 11,75 & 109 & 70 \\
AM3 & 79.3 & 12,04 & 100 & 70 \\
AM4 & 93.8 & 13,23 & 109 & 70 \\
\hline
\end{tabular}




\begin{tabular}{lcccc}
\hline AM5 & 88.4 & 12,79 & 101 & 70 \\
AM6 & 109 & 0 & 109 & 109 \\
\hline
\end{tabular}

Tabela 3. Distribuição da frequência da EMTI das 10 idosas com doença de Alzheimer

\begin{tabular}{ccc}
\hline CLASSIFICAÇÃO & N & \% \\
\hline Normal médio & 6 & 60,0 \\
Normal baixo & 3 & 30,0 \\
Inferior & 1 & 10,0 \\
\hline
\end{tabular}

Fonte: Pesquisa de Campo, 2013.

Considerando a pontuação total das participantes, foi possível traçar o perfil motor das idosas, como se vê na Tabela 3.

\section{DISCUSSÃO}

A doença de Alzheimer por apresentar maiores comprometimentos de memória e percepção do corpo, e sendo a cognição destes a mais afetada acaba sendo o principal foco de estudos de diversos pesquisadores. Vários dados bibliográficos podem ser encontrados a respeito de tal assunto. Porém, este comprometimento pode acarretar diversas complicações motoras, por este motivoa presente pesquisa objetivou avaliar o desempenho cognitivo e motor em idosas com doença de Alzheimer.

As características das pesquisadas deste estudo foram 10 idosas com a média de idade de 78,8 anos com idade mínima de 65 e máxima de 83 anos, casadas, e com nível inferior fundamental incompleto, dados próximos aos encontrados por Luzardoet al
(2006), no qual demonstrou que a média de idade dos idosos foi de 75,19, com idade variando entre 61 e 86 anos, sendo que as maiorias dos idosos eram do sexo feminino em $66,7 \%$ dos casos, com estado civil casados, com escolaridade variando em torno de quatro anos.

Colaborando com a ideia, Machado et al (2009)conta em seu estudo que o impacto da demência causa vários fatores de risco associados, tais como: natureza da doença marcadamente dependente da idade, que caracteriza a deficiência cognitiva, a predominância do sexo feminino, a acentuada importância dos fatores socioeconômicos e associação com a incapacidade de desempenhar as atividades cotidianas. Informações compatíveis com o presente estudo no local pesquisado, só havia mulheres 
com a doença e baixa escolaridade, mas sem grandes dificuldades para desempenhar as atividades diárias.

Analisando os dados da avaliação por meio do MEEM, verificou-se que a média geralfoi 19,8 pontos, dados que demonstram possível declínio da função cognitiva, uma vez que o resultado ficou próximo ao valor esperado para um individuo analfabeto (20 pontos).Quatro idosas que estão no estágio intermediário da doença apresentavam escore abaixo do normal que variou entre 12 e 18 pontos, sendo assim, acredita-se que com o avançar da idade e da doença maior $o$ comprometimento da função cognitiva.

Quanto a avaliação da aptidão motora através da EMTI, existem poucas referencias quanto ao assunto direcionado a população de doença de Alzheimer, o que dificultou, em partes, a realização da discussão do trabalho. Todavia, pode-se observar que a doença traz prejuízos de acordo com a evolução da doença e também com a presença ou não de estímulos motores.

Durante avaliação de atividade de motricidade fina (AM1), as idosas não apresentaram muita dificuldade, em virtude de realizar atividades constantes/regulares na instituição, frequentemente são estimuladas a trabalhar atividades como pintura, colagem, bordado, etc.

$\mathrm{Na}$ motricidade global (AM2), as idosas tiveram um pouco de dificuldade para realizar as atividades, pois para que esses movimentos sejam bem sucedidos dependem de coordenação motora, precisão, ritmo, agilidade, fluidez, postura e um bom tônus muscular. Colaborando com a ideia de Rosa Neto et al(2011)que mostra em sua pesquisa que a (AM2) os participantes mostraram maior grau de dificuldade. Esta área é responsável pela regulação do equilíbrio e da atitude do indivíduo, desempenhando um importante papel na melhora dos comandos nervosos e no afinamento das percepções e sensações.

Nas tarefas avaliadas através de movimento de estabilidade e propriocepção (AM3), as idosas tiveram dificuldade de realizar as atividades, e obtiveram resultados inferiores dentre o perfil de escala motora. $\mathrm{O}$ que pode se explicar pelo fato das idosas nem sempre aderirem às atividades motoras, ou mesmo pela diminuição fisiológica da manutenção de equilíbrio intensificado pela doença. De acordo com a ideia de Monteiro (2012), destaca que o equilíbrio é a base primordial de toda ação diferenciada dos segmentos corporais. Quanto mais é defeituoso o movimento, mais energia consome e a postura é a atividade reflexa do corpo com relação ao espaço.

Já na atividade de esquema corporal (AM4) as idosas não apresentaram dificuldade para realizar a tarefa, pois se pressupõem que uma boa evolução da motricidade e percepções espaciais e da afetividade, influenciou no resultado do teste. 
Concordando com Fonseca et al (2012), que o desgaste natural do organismo, característico do idoso, provoca perda na multiplicidade do sistema sensorial e altera o processo de fornecimento de informações sobre a percepção corporal. $O$ que reforça a explicação do estímulo que estas idosas recebem na instituição, no qual vem retardando a perda desta função importante durante o processo de envelhecimento.

Nas atividades de organização espacial (AM5), algumas idosas tiveram dificuldade em realizar a atividade, pois não tinham percepção de espaço e de identificar onde e em qual local poderiam ou não se instalar. Já na atividade de organização temporal (AM6), todas as idosas tiveram um bom aproveitamento da atividade, pois conseguiram identificar lembranças passada, o que mostra que o indivíduo tem maior facilidade de recordar de momentos passados do que presente.

Ao final da avaliação motora, a maioria das pesquisadas $(60 \%)$ foi classificada como rendimento normal médio, ou seja, conseguem realizar as tarefas solicitadas de coordenação grossa e fina, equilíbrio, etc., porém começam a apresentar certa dificuldade ou lentidão para conclusão da mesma. E apenas $10 \%$ das idosas foi classificada como rendimento inferior, com maiores dificuldades ou impossibilidade de realização das tarefas.
Zidanet al (2012) em seu estudo, identificou que o declínio motor ocorre de forma linear, piorando com o maior comprometimento da doença.

Sob esta ótica, neste estudo foi observado que a maioria das idosas encontrase em classificação de normal médio, talvez em virtude das participantes encontrarem-se no estágio inicial da doença e, que não tiveram resultados superiores, devido ao fator limitante da doença, e também com o envelhecimento, o equilíbrio pode ser prejudicado em razão dos problemas físicos, sedentarismo, neurológicos e emocionais. Concordando com a afirmação de Rosa Netoet al (2011), de que certas exigências da tarefa podem sobrecarregar demasiadamente os sistemas neurofisiológicos que se deterioraram com o envelhecimento.

Hernandez et al (2010) observou em seu estudo que idosos com comprometimento cognitivo leve apresentam diminuição de equilíbrio e coordenação e diminuição nos níveis de atividade física, aumentando o risco de lesões, quedas e fraturas. Em base desta citação, o estudo encontrou idosas com comprometimento moderado, já estariam mais pré-dispostas aos fatores de risco, porém como a instituição promove estímulos cognitivos e motores, essas senhoras estão com rendimento motor relativamente preservado, diminuindo ou retardando os fatores de risco associados à doença. 


\section{CONCLUSÃO}

A pesquisa não identificou comprometimento motor e cognitivo avançados nas idosas pesquisadas. Entretanto, a qualidade de vida também é associada a um bom desempenho motor,o estímulo cognitivo e motor que as idosas recebem na instituição mostraram-se eficaz no controle e atenuação dos níveis de aptidão motora.

\section{REFERÊNCIAS}

ABREU, I. D. et al. Demência de alzheimer: correlação entre memória e autonomia. Revista de Psiquiatria Clínica. Minas Gerais, v 32, fevereiro de 2005. Disponível em:

http://www.scielo.br/pdf/rpc/v32n3/a05v32n3 .pdf

APRAHAMIAN, I.; MARTINELLI, J.E.; YASSUDA, M.S. Doenca de Alzheimer: revisão da epidemiologia e diagnostico. Rev Bras Clin Med, 2009;7:27-35.

FERRERI, F.; PAURI, F.; PASQUALETTI, P.; FINI, R.; DAL FORNO, G.; ROSSINI, M. Motor Cortex Excitability in Alzheimer`s Disease: A Transcranial Magnetic Stimulation Study.Disponivelem: http://www.ncbi.nlm.nih.gov/pubmed/125098 $\underline{53}$

FONSECA, C.C. et al. Benefícios da estimulação perceptual corporal no esquema corporal de idosos. Rev. bras. geriatr. gerontol. vol.15 no.2 Rio de Janeiro 2012. Disponível em:

http://www.scielo.br/scielo.php?script=sci_art text\&pid=S1809-98232012000200017

GALLO, J. et al. Assistência ao idoso, aspecto clinico do envelhecimento. Rio de Janeiro. $5^{\circ}$ ed. Guanabara Koogan, 2001.
A literatura frequentemente aborda a DA e seu comprometimento cognitivo, não chamando atenção para o declínio motor da doença que também pode ser bastante limitante para as idosas. Em virtude disto, sugerem-se novas pesquisas voltadas para a investigação do comprometimento motor em Alzheimer, com maior $\mathrm{n}$ amostral e também variados instrumentos de avaliação.

HERNANDEZ, S.S.S.; COELHO, M.F.G.; GOBBI, S.; STELLA, F. Efeitos de um programa de atividade física nas funções cognitivas, equilíbrio e risco de quedas em idosos com demência de Alzheimer. Revista de Brasileira Fisioterapia, São Carlos, v.14, n.1, p.68-74, jan./fev. 2010. Disponível em:http://www.scielo.br/pdf/rbfis/v14n1/11.p $\underline{\mathrm{df}}$

LUCCHETTI et al. O idoso e sua espiritualidade. Revista Brasileira de Geriatria e Gerontologia.Rio de Janeiro, v.14 n.1, janeiro de 2011. Disponível em:http://revista.unati.uerj.br/scielo.php?scrip $\mathrm{t}=$ sci_arttext\&pid=S18099823201100010001 $\underline{6 \& \operatorname{lng}=\mathrm{pt} \& \mathrm{nrm}=\mathrm{iso}}$

LUZARDO, R.R; GORINI, C.P.I.M; SILVA, S.S.P.A;Características de Idosos com Doença de Alzheimer e seus Cuidadores. Florianópolis, 2006 Out-Dez; 15(4): 587-94. Disponível em http://www.scielo.br/pdf/tce/v15n4/v15n4a06. $\underline{\mathrm{pdf}}$

MACHADO, C, J; RIBEIRO,L. C.R; COTTA, M.M.R; LEAL, G.F.P;Declínio Cognitivo de Idosos e sua Associação com Fatores Epidemiológicos em Viçosa. Revista Brasisleira de Geriatria e Gerontologia, v.14 n.1Rio de Janeiro 2011. Disponível em:http://revista.unati.uerj.br/scielo.php?scrip 
$\mathrm{t}=$ sci_arttext $\&$ pid=S180998232011000100012\&lng=pt\&nrm=iso MEDEIROS E RO. Tradução, adaptação cultural e análise das propriedades psicométricas do Activitiesof Daily Living Questionnaire (ADLQ) para avaliação funcional de pacientes com a doença de Alzheimer. Revista Brasileira de Fisioterapia. São Carlos, v 13 , Junho de 2009 . Disponível em:http://www.scielo.br/scielo.php?pid=S141 $\underline{335552009000300011 \& \text { script }=\text { sci_arttext }}$

\section{MONTEIRO, M. M. L. Psicomotrocidade e} qualidade de vida em idosos institucionalizados.Pro- reitoria de pos graduação pesquisa stricto sensu em mestrado em gerontologia, Brasília, 2012. Disponível em:http://www.bdtd.ucb.br/tede/tde_arquivos/ 17/TDE-2012-11-12T065815Z-

1365/Publico/Michelly\%20Mara\%20Lira\%20 Monteiro.pdf

PERRACINI,M.R.;FLO,C.M. Funcionalidade e Envelhecimento. Rio de Janeiro: Guanabara Koogan, 2009.

ROSA NETO, F. et al. Parâmetros motores dos Parkinsonianos da região conurbada de Florianópolis. Revista Brasileira de Ciência e Movimento. v. 12, n. 1Brasília, março de 2004. Disponível em:

http://portalrevistas.ucb.br/index.php/RBCM/ article/view/536

ROSA NETO F, SAKAE TM E POETA LS. Validação dos parâmetros motores na terceira idade. Revista Brasileira de Ciência e Movimento, 2011;19(1):20-25. Disponível em:

http://portalrevistas.ucb.br/index.php/RBCM/ article/viewFile/1837/1847

ZAGO, A. S.; GOBBI S. Valores normativos da aptidão funcional de mulheres de 60 a 70 anos.Revista Brasileira de Ciência e Movimento. Brasíliav. 11 n. 2, Junho de 2003. Disponível em:

http://www.contag.org.br/imagens/f1858valor es-normativos-da-aptidao-funcional-de$\underline{\text { mulheres-de-60-a-70-anos.pdf }}$
ZIDAN, M.; ARCOVERDE, C.; ARAUJO, N.B.; VASQUES, P.; RIOS, A.; LAKS, J.; DESLANDES, A. Alterações motoras e funcionais em diferentes estágios da doença de Alzheimer. RevPsiqClin. 2012;39(5):1615 . 